SECTION 31. Economic research, finance, innovation.

Naumov Anatoly Aleksandrovich

Docent, candidate of Technical Sciences, Center of Applied Mathematical Research, Novosibirsk, Russia, E-mail: A_A_Naumov@mail.ru

\title{
REDISTRIBUTION OF TOTAL INCOME BETWEEN OF INTEGRATED PROJECT PARTICIPANTS
}

The paper discusses methods for estimating the effectiveness of integrated projects based on the procedure of compounding financial flows. In particular proposed new scheme for calculating the NFV index for projects. These schemes are based on the method detailed flow. The new indicator was called as $N F V_{D F}$ (Net Future Value for Disintegration Flows). The use of detailed flow analysis allows the problem of integrated investment projects analysis to their practical using.

Keywords: Integrated investment projects, efficiency, NFV criterion, the method of detailed flows.

\section{УДК 330.46:330.322.5: 658.155}

\section{ПЕРЕРАСПРЕДЕЛЕНИЕ ОБЩЕГО ДОХОДА МЕЖДУ УЧАСТНИКАМИ ИНТЕГРИРОВАННОГО ПРОЕКТА}

В работе рассмотрень методы оченивания эффективности интегрированных проектов на основе процедуры компаундирования финансовых потоков. В частности, предложены новые схемы расчета показателя NFV для частных проектов. Эти схемы основаны на методе детализачии потоков [1]. Новый показатель назван $N F V_{D F}$ (Net Future Value for Disintegration Flows). Использование метода детализации потоков позволяет приблизить задачу анализа интегрированных инвестиционных проектов к их практическому использованию.

Ключевые слова: интегрированные инвестиционные проекты, эффрективность, NFV критерий, метод детализации потоков.

Постановка задачи. Пусть для каждого из $\mathrm{n}$ частных проектов $\mathrm{Pr}_{\mathrm{i}}$, $\mathrm{i}=1,2, \ldots, \mathrm{n}$, в рамках интегрированного проекта $\operatorname{Pr}$ известны входные и выходные потоки в виде: $\mathrm{F}_{\mathrm{in},(\mathrm{i})}(\mathrm{t}), \mathrm{t}=\mathrm{t}_{0}, \mathrm{t}_{1}, \mathrm{t}_{2}, \ldots,\left(\mathrm{t}_{\mathrm{m}}=\mathrm{T}\right), \mathrm{i}=1,2, \ldots, \mathrm{n},-$ входные финансовые потоки (инвестиций, вложений), $\mathrm{F}_{\text {out,(i) }}(\mathrm{t}), \mathrm{t}=$ $\mathrm{t}_{0}, \mathrm{t}_{1}, \mathrm{t}_{2}, \ldots,\left(\mathrm{t}_{\mathrm{m}}=\mathrm{T}\right), \mathrm{i}=1,2, \ldots, \mathrm{n}, \quad$ - выходные финансовые потоки (доходов). Для простоты записи формул при расчете показателей 
эффективности проектов, будем предполагать, что моменты времени $\mathrm{t}_{0}, \mathrm{t}_{1}, \mathrm{t}_{2}, \ldots,\left(\mathrm{t}_{\mathrm{m}}=\mathrm{T}\right)$, - положительные целые числа и все интервалы между соседними отсчетами равны единице времени. Потоки разных проектов могут накладываться (перекрываться) во времени, но каждый из проектов участвует в интегрированном проекте своими потоками только на одном из временных интервалов $-\left[\mathrm{t}_{0,(\mathrm{i})} ; \mathrm{T}_{(\mathrm{i})}\right] \subseteq\left[\mathrm{t}_{0} ; \mathrm{T}\right], \mathrm{i}=1,2, \ldots, \mathrm{n}$. Требуется оценить эффективность интегрированного проекта и каждого из частных проектов.

Оценивание эффективности интегрированного проекта. Проведем детализацию входного $\left(\mathrm{F}_{\mathrm{in}}(\mathrm{t}), \mathrm{t}=\mathrm{t}_{0}, \mathrm{t}_{1}, \mathrm{t}_{2}, \ldots, \mathrm{t}_{\mathrm{m}}=\mathrm{T}\right)$ и выходного $\left(\mathrm{F}_{\text {out }}(\mathrm{t}), \mathrm{t}=\mathrm{t}_{0}, \mathrm{t}_{1}, \mathrm{t}_{2}, \ldots, \mathrm{t}_{\mathrm{m}}=\mathrm{T}\right.$ ) потоков проекта $\operatorname{Pr}$ (см. [1], [2]). Отметим, что, в отличие от детализации потоков неинтегрированных проектов, в случае интегрированных проектов увеличивается число вариантов для параметров детализации, поскольку в этом случае помимо использования заемных средств общепринятых внешних источников заимствований (банков, компаний, проектов и пр.) можно воспользоваться средствами каждого из участников интеграции. Однако следует отметить, что увеличение сложности структуры и взаимодействий интегрированного проекта приводит к возрастанию его рисков.

Процедура детализации элементов входного потока (уточнение характеристик источников финансирования проекта) выполняется для моментов времени $\mathrm{t}=\mathrm{t}_{0}, \mathrm{t}_{1}, \mathrm{t}_{2}, \ldots, \mathrm{t}_{\mathrm{m}}$, до момента времени $\mathrm{t}=\mathrm{t}_{\mathrm{m}}$ включительно (при этом получим векторы $\mathrm{F}_{\mathrm{in}, \mathrm{DF}, \mathrm{m},(\mathrm{i})}$, $\mathrm{i} \in \mathrm{N}_{\mathrm{t}_{\mathrm{m}}}^{+}$), а затем

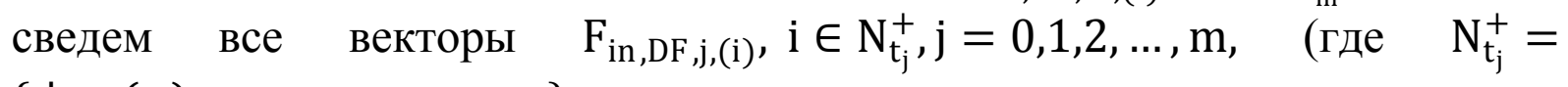
$\left.\left\{\mathrm{i} \mid \operatorname{Pr}_{\mathrm{i}}\left(\mathrm{t}_{\mathrm{j}}\right)>0, i=1,2, \ldots, n\right\}, \mathrm{j}=0,1,2, \ldots, \mathrm{m}\right)$ в один общий вектор с учетом привязки компонент этих векторов к моментам времени и суммируя значения векторов относящихся к одним и тем же моментам времени. Очевидно, в общем случае число компонент в новом свернутом векторе будет больше общего числа временных отсчетов $(\mathrm{m}+1)$ интегрированного проекта Pr. Это означает, что элементы входного потока, относящиеся к моментам времени следующими за моментом $\mathrm{t}_{\mathrm{m}}$, будут погашаться за пределами горизонта рассмотрения потоков проекта, т.е. за границами временного интервала $\left[\mathrm{t}_{0} ; \mathrm{T}\right]$. Рассмотрение тонкостей расчетных схем для таких случаев оставим для последующих исследований.

В результате свертки векторов $\mathrm{F}_{\mathrm{in}, \mathrm{DF}, \mathrm{j}, \mathrm{i})}, \mathrm{i} \in \mathrm{N}_{\mathrm{t}_{\mathrm{j}}}^{+}, \mathrm{j}=0,1,2, \ldots, \mathrm{m}$, получим новый вектор $\mathrm{F}_{\mathrm{in}, \mathrm{DF}}^{\Sigma}$. Условно свертку векторов обозначим

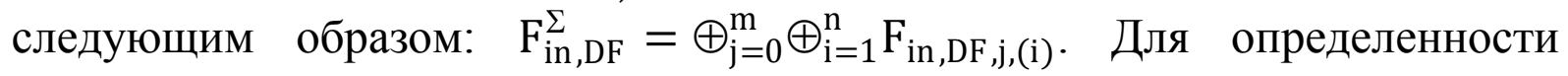
положим, что компоненты вектора $\mathrm{F}_{\mathrm{in}, \mathrm{DF}}^{\Sigma}$ относятся к моментам времени $\mathrm{t}=\mathrm{t}_{0}, \mathrm{t}_{1}, \mathrm{t}_{2}, \ldots, \mathrm{t}_{\mathrm{DF}}=\mathrm{T}_{\mathrm{DF}}$ и, как было отмечено выше, в общем случае выполняется неравенство $\mathrm{T}_{\mathrm{DF}} \geq \mathrm{T}$. 
Переходим к детализации элементов выходного потока проекта $\mathrm{F}_{\text {out }}(\mathrm{t})=\sum_{\mathrm{i}=1}^{\mathrm{n}} \mathrm{F}_{\text {out,(i) }}(\mathrm{t}), \mathrm{t}=\mathrm{t}_{0}, \mathrm{t}_{1}, \mathrm{t}_{2}, \ldots, \mathrm{t}_{\mathrm{m}}$. Напомним, что $\mathrm{F}_{\text {out,(i) }}(\mathrm{t})$ элементы выходного потока проекта $\operatorname{Pr}_{\mathrm{i}}, \mathrm{i}=1,2, \ldots, \mathrm{n}$. Для этого требуется расписать (распределить) элементы этого потока в два потока: один будет показывать, как компенсируются (погашаются) элементы потока $\mathrm{F}_{\mathrm{in,DF}}^{\Sigma}$ (обозначим его через $\mathrm{F}_{\text {out,IP }}^{\Sigma}$, где IP - Internal Projects) и второй - поток, который может быть выведен из проекта $\operatorname{Pr}$ и использован в других проектах (обозначим как $\mathrm{F}_{\text {out,EP }}^{\Sigma}$, где EP - External Projects).

Как и для элементов входного потока, в данном случае можно записать свертки: $\mathrm{F}_{\text {out }, \mathrm{IP}}^{\Sigma}=\oplus_{\mathrm{j}=0}^{\mathrm{m}} \oplus_{\mathrm{i}=1}^{\mathrm{n}} \mathrm{F}_{\text {out }, \mathrm{IP}, \mathrm{j},(\mathrm{i})}$ и $\quad \mathrm{F}_{\text {out }}=\mathrm{F}_{\text {out }, \mathrm{IP}}^{\Sigma} \oplus \mathrm{F}_{\text {out }, \mathrm{EP}}^{\Sigma}$. Здесь $\quad \mathrm{F}_{\text {out }}=\left(\mathrm{F}_{\text {out }}\left(\mathrm{t}_{0}\right), \mathrm{F}_{\text {out }}\left(\mathrm{t}_{1}\right), \ldots, \mathrm{F}_{\text {out }}\left(\mathrm{t}_{\mathrm{m}}\right)\right)$. Заметим также, что выполняются равенства $\mathrm{F}_{\text {out,IP,j,(i) }}=\mathrm{F}_{\mathrm{in,DF}, \mathrm{j},(\mathrm{i})}$ для всех $\mathrm{F}_{\mathrm{in,DF}, \mathrm{j},(\mathrm{i})}>0$, $\mathrm{i} \in \mathrm{N}_{\mathrm{t}_{\mathrm{j}}}^{+}, \mathrm{j}=0,1,2, \ldots, \mathrm{m}$. Эти равенства имеют понятный смысл: расходы на погашение кредитов и инвестиции из собственных средств для каждого частного проекта $\operatorname{Pr}_{\mathrm{i}}, \mathrm{i}=1,2, \ldots, \mathrm{n}$, должны браться из общих доходов интегрированного проекта $\mathrm{Pr}$. Таким образом, элементы вектора $\mathrm{F}_{\text {out,IP }}^{\Sigma}$ должны полностью покрывать компоненты (совпадать с компонентами, быть равны компонентам) вектора $\mathrm{F}_{\mathrm{in}, \mathrm{DF}}^{\Sigma}$ для всех моментов времени $\mathrm{t}=\mathrm{t}_{0}, \mathrm{t}_{1}, \mathrm{t}_{2}, \ldots, \mathrm{t}_{\mathrm{m}}$. Формально это можно записать таким образом: $\mathrm{F}_{\text {out }, \mathrm{IP}}^{\Sigma}(\mathrm{t})=\mathrm{F}_{\mathrm{in}, \mathrm{DF}}^{\Sigma}(\mathrm{t})$ для всех $\mathrm{t} \in\left\{\mathrm{t}_{0}, \mathrm{t}_{1}, \mathrm{t}_{2}, \ldots, \mathrm{t}_{\mathrm{m}}\right\}$. Если это условие не выполняется, то необходимо повторить процедуру детализации (изменив источники кредитования, условия кредитования и т.д.) или сделать вывод о том, что проект Pr не является эффективным. Последнее означает, что его выходной поток (доходов) не может компенсировать входной поток (инвестиций). Если равенства $\mathrm{F}_{\text {out,IP }}^{\Sigma}(\mathrm{t})=\mathrm{F}_{\mathrm{in,DF}}^{\Sigma}(\mathrm{t})$ для всех $\mathrm{t} \in$ $\left\{\mathrm{t}_{0}, \mathrm{t}_{1}, \mathrm{t}_{2}, \ldots, \mathrm{t}_{\mathrm{m}}\right\}$ выполнились, то можно перейти к оцениванию показателя эффективности (будущего дохода) интегрированного проекта $\mathrm{NFV}_{\mathrm{I}}$ в соответствии с формулой: $\mathrm{NFV}_{\mathrm{I}}=\sum_{\mathrm{t}} \mathrm{F}_{\text {out }, \mathrm{EP}}(\mathrm{t}) \cdot\left(1+\mathrm{r}_{\text {out,EP }}(\mathrm{t})\right)^{\mathrm{T}-\mathrm{t}}$. Здесь $\mathrm{F}_{\text {out,EP }}^{\Sigma}(\mathrm{t})$ - элемент вектора $\mathrm{F}_{\text {out,EP }}^{\Sigma}$, относящийся к моменту времени $\mathrm{t}$, $\mathrm{r}_{\text {out,EP }}(\mathrm{t})$ - ставка внешнего использования (во внешних по отношению к проекту $\operatorname{Pr}$ проектах) средств в размере $\mathrm{F}_{\text {out,EP }}^{\Sigma}(\mathrm{t})$. Заметим, что в общем случае таких ставок может быть несколько, и они соответствуют доходностям проектов, в которые вкладываются средства $\mathrm{F}_{\text {out,EP }}^{\Sigma}(\mathrm{t})$. Для оценивания доходности проекта $\operatorname{Pr}$ можно воспользоваться формулой: $\mathrm{IRR}_{\mathrm{DF}, \mathrm{in}, \mathrm{NFV}+\mathrm{DF}}=\left\{\mathrm{r} \mid \sum_{\mathrm{t}} \mathrm{F}_{\mathrm{in}}(\mathrm{t}) \cdot(1+\mathrm{r})^{\mathrm{T}-\mathrm{t}}=\mathrm{NFV}_{\mathrm{I}}+\sum_{\mathrm{t}} \mathrm{F}_{\mathrm{in}, \mathrm{DF}}^{\Sigma}(\mathrm{t})\right\}$.

Остановимся подробнее на методе оценивания доходов каждого из частных проектов $\operatorname{Pr}_{\mathrm{i}}, \mathrm{i}=1,2, \ldots, \mathrm{n}$. Оценить эти доходы - это значит найти те части общего дохода $\mathrm{NFV}_{\mathrm{I}}$, которые приходятся на каждый из частных проектов. Так, например, можно воспользоваться идеей оценивания дохода для каждого из частных проектов $\mathrm{Pr}_{\mathrm{i}}, \mathrm{i}=1,2, \ldots, \mathrm{n}$, через определение 
долей от общего дохода интегрированного проекта $\mathrm{NFV}_{\mathrm{I}}$, приходящихся на каждый из этих проектов. Для этого воспользуемся формулами:

$$
\begin{aligned}
& \operatorname{Profit}_{(\mathrm{i})}=\sum_{\mathrm{t}} \mathrm{F}_{\mathrm{in},(\mathrm{i})}(\mathrm{t}) \cdot\left(1+\mathrm{IRR}^{\mathrm{T}-\mathrm{t}}, \mathrm{i}=1,2, \ldots, \mathrm{n},\right. \\
& \mathrm{NFV}_{(\mathrm{i})}=\mathrm{NFV}_{\mathrm{I}} \cdot \operatorname{Profit}_{(\mathrm{i})} / \sum_{\mathrm{i}=1}^{\mathrm{n}} \operatorname{Profit}_{(\mathrm{i})}, \mathrm{i}=1,2, \ldots, \mathrm{n} .
\end{aligned}
$$

Здесь $\operatorname{Profit}_{(\mathrm{i})} / \sum_{\mathrm{i}=1}^{\mathrm{n}} \operatorname{Profit}_{(\mathrm{i})}, \mathrm{i}=1,2, \ldots, \mathrm{n},-$ доли от общего дохода для каждого из частных проектов, IRR - доходность проекта Pr. Найдем доходности каждого из проектов в соответствии с их доходами: $\operatorname{IRR}_{(\mathrm{i})}=$ $\left\{\mathrm{r} \mid \sum_{\mathrm{t}} \mathrm{F}_{\mathrm{in},(\mathrm{i})}(\mathrm{t}) \cdot(1+\mathrm{r})^{\mathrm{T}-\mathrm{t}}=\mathrm{NFV}_{(\mathrm{i})}\right\}, \mathrm{i}=1,2, \ldots, \mathrm{n}$.

\section{Литература}

1. Наумов А.А. Модификация критерия $N F V$ на основе метода детализации финансовых потоков проектов/ А.A. Наумов// Theoretical\&Applied Science, Materials of the ISPC «Results \&Perspectives», 30.09.2013, Florence, Italy, 2013, № 9 (5), C. 105-109.

2. Список трудов [Электронный ресурс]. URL: https://sites.google.com/site/ anatolynaumov2011/home/spisok-trudov-list-of-papers (дата обращения: 25.09.2013). 